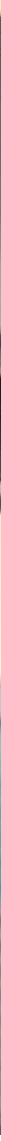

Researchers such as Anne-Julie Chabot-Doré, pictured in Luda Diatchenko's lab at McGill University in Montreal, Canada, are searching for chronic pain genes.

GENETICS

\title{
An incomplete mosaic
}

\section{Although genetics studies have so far failed to revolutionize pain treatments, some researchers think that a host of discoveries are just around the corner.}

\section{BY ERYN BROWN}

$\mathrm{N}$ eurologist Stephen Waxman wants to understand how genes influence chronic pain. He hopes that unravelling the mystery will offer relief to the patients he studies. Some are in agony because of old injuries, others because of diabetic nerve damage. And there are those who battle rare disorders such as inherited erythromelalgia, experiencing searing pain in their extremities when they come into contact with mild warmth or engage in moderate exercise. "For these people, putting on socks is like having hot lava poured on their bodies," Waxman says.

Researchers such as Waxman, who is at Yale University's Center for Neuroscience and Regeneration Research in West Haven, Connecticut, are betting that analysing patients' DNA will help to explain the underlying causes and mechanics of chronic pain, which now afflicts around one billion people worldwide. Ultimately, researchers hope that such explorations will lead to better treatments for those who have chronic pain, by revealing targets for a new generation of drugs that are capable of targeting pain without dulling the senses or promoting addiction.

But this is a search that has already gone on for a couple of decades. And although scientists have discovered that genetics have a significant role in pain - anywhere from around $20 \%$ to $60 \%$ of the variability in how people experience pain is attributable to differences in genes no one has yet pinpointed any crucial smoking guns in DNA. And the complexity of the problem can be a bit depressing. "Once you realize something is mediated by, say, 1,000 genes, you wonder if it's even possible to figure it out," says Jeffrey Mogil, a pain researcher at McGill University in Montreal, Canada. But other researchers are more optimistic, and suggest that continuing with the approach, with some refinements, will yield useful discoveries.

\section{ELEMENTAL CONCERNS}

Across the campus from Mogil's office, molecular geneticist Luda Diatchenko is undeterred in her search for pain genes. Diatchenko thinks that the large number of unknowns means that scientists are actually on the verge of a flood of discoveries. It took time, she says, to design pain-genetics experiments and to develop methods for studying the genome that were not prohibitively expensive. "The studies have not been done yet," Diatchenko says. "It will be an explosion — soon."

Pain-genetics researchers have pursued two main avenues of inquiry, says neuroscientist Stephen McMahon at King's College London. The first strategy, taken by researchers such as Waxman, is to study rare pain disorders that run in families to identify single-gene mutations. This approach has produced a handful of tantalizing leads. For instance, inherited erythromelalgia, which Waxman says affects around 30-40 families worldwide, is caused by a mutation in a gene that causes the sodiumion channel $\mathrm{Na}_{\mathrm{V}} 1.7$ to become overactive. This protein is crucial for conducting pain signals in peripheral nerves, but not, it is thought, in the central nervous system.

If drug developers could use this information to come up with a way to dial down $\mathrm{Na}_{\mathrm{V}} 1.7$ in people with chronic pain, they could develop systemic treatments that would dampen pain signals in nerve cells without causing side effects such as sleepiness, confusion, loss of balance or addiction, says Waxman. Drug companies such as Amgen, Pfizer and Convergence Pharmaceuticals are 
working on $\mathrm{Na}_{\mathrm{V}} 1.7$ inhibitors, but Waxman doesn't expect to see any therapies approved for several years. "Finding a drug that's selective for this sodium channel is tricky," says McMahon. And accidentally hitting another subtype of sodium channel - such as the ones that are essential for controlling heartbeat - would be dangerous.

The second approach is broader: rather than just looking at rare inherited conditions, the DNA from large cohorts of patients is sequenced to try to identify genetic variants and the traits, or phenotypes, that correlate with them. The hope was that such studies would reveal a small number of key pain genes - those shared by all or many people with various chronic pain disorders. But what researchers found instead was that pain, like many chronic conditions, is caused by a complex interaction between genes and the environment, influenced by hundreds, if not thousands, of genes in each individual.

William Maixner, director of the Center for Translational Pain Medicine at Duke University in Durham, North Carolina, refers to the causes of chronic pain as "a mosaic of pathways" within each individual that change over time owing to environmental factors, and that affect psychological processes as well as those related to nerve damage. Maixner is working with Diatchenko and others to tease out the genetic mechanisms that are at work in a number of pain disorders, including irritable bowel syndrome, fibromyalgia and lower back pain.

Their Orofacial Pain: Prospective Evaluation and Risk Assessment, or OPPERA, study focuses on people with temporomandibular disorder (TMD), a common facial-pain condition of unclear origin. The team collected data for up to 5 years from just over 2,700 TMDfree men and women, 260 of whom developed TMD during the study. Maixner's group examined DNA variations known as single nucleotide polymorphisms (SNPs) in 358 genes that regulate pain, and tracked 202 phenotypes in the volunteers ${ }^{1}$. The initial findings underscored the complexity of TMD. The research-

"The studies have not been done yet. It will be an explosion soon."

ers failed to identify any

single genetic variation associated with the condition, but did find five SNPs linked with risk factors for TMD, including ones related to non-specific facial pain, physical symptoms, stress and negative mood. The team has since expanded its analysis to the entire genome of the OPPERA participants, and hopes to publish updated results by 2017 .

Researchers such as Mogil and McMahon, who are vexed by the slow progress in pain genomics, say that genome-wide association studies have been too small to detect culprit variants - and that the funding isn't there to

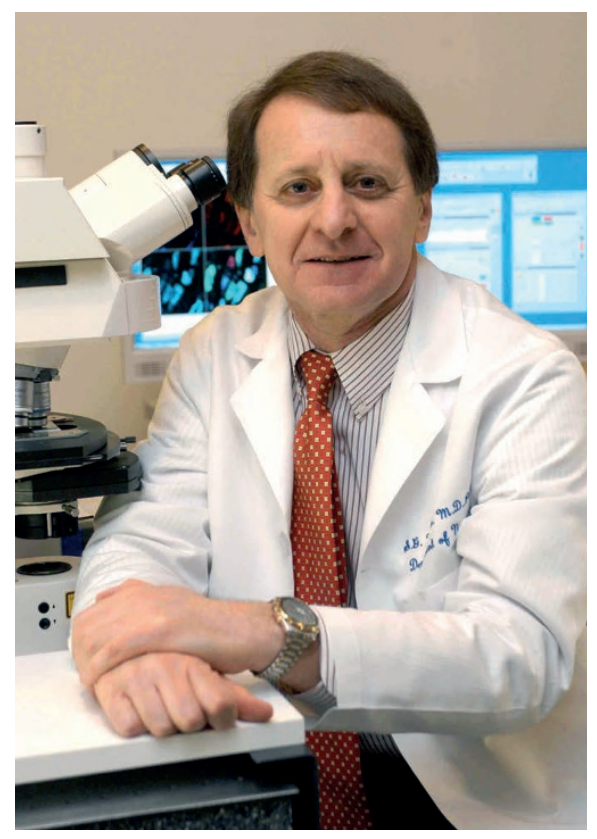

Neurologist Stephen Waxman studies the genetics of rare pain disorders.

support larger-scale efforts.

Compounding the problem is the difficulty of correctly phenotyping people with chronic pain. Christopher Sivert Nielsen, a pain psychologist at the Norwegian Institute of Public Health in Oslo, says that pain disorders that receive separate diagnoses are often not that different from each other. Furthermore, chronic pain is very common, making the identification of controls challenging. In this sense, chronic pain is very different from well-characterized diseases such as multiple sclerosis (MS). "If you study MS, you go into the clinic, you collect cases and the rest of the world provides your controls," Nielsen says. "But this doesn't work for pain." Because many types of pain have common genetic origins, it's easy for a person with a related pain type to end up in a control group, disrupting an association study. To pin down phenotypes, Nielsen adds, researchers will need to screen study participants much more rigorously - a difficult task in the large groups required to do genome-wide association studies well.

Maixner says that OPPERA researchers are trying to cut through the noise in their data by devising new methodologies to understand how genes relate to symptoms. Bioinformaticians are working on stratification procedures that divide the study population into three distinct subgroups - a pain-sensitive cluster of people with heightened sensitivity to experimental pain stimuli, a global-symptoms cluster with pain sensitivity and psychological distress, and a third group with neither ${ }^{2}$. Most people with TMD fall into the first and second groups. By analysing these clusters instead of the entire population, Maixner suggests that scientists will find it easier to tease out how genes contribute to the development and to the manifestation of symptoms in each group.

Some researchers are looking beyond the genome to the epigenome - the markers on DNA that have been added by processes such as methylation. Epigenetic changes alter gene expression and therefore affect various biochemical events. "The hope is to discover a whole new number of cellular processes that control the dynamics of a chronic pain state - the 'on' switches," says McMahon.

He says that studying epigenetics could help scientists to understand the environmental influences that make a person more likely to develop chronic pain. He thinks it could also improve treatments, reasoning that drugs that interfere higher up the chain of biological events should prove more effective than therapies that operate at the periphery. "Using $\mathrm{Na}_{\mathrm{V}}$ blockers is like attacking the foot soldiers, whereas disrupting epigenetic processes is like taking out a general giving an order to the whole army," he says.

In 2014, McMahon collaborated on a study that looked at identical twins with different levels of pain sensitivity. The team found ${ }^{3}$ methylation differences connected to several genes, including the pain gene TRPA1. But epigenetics is dizzyingly complex, and other researchers note that it is not yet possible to link epigenetic changes to the environmental factors that might have caused them. "It's early days," concedes McMahon, who stresses that merely finding epigenetic or genetic targets isn't enough - such work must be accompanied by experiments in cells and model organisms that explain the biology going on in the cells.

And Mogil wonders if "epigenetics is a bit of a flavour of the month". Genetics failed, so researchers are now asking "Where will we bet all our chips now?" he says. For now, Mogil has largely shifted the focus of his research from identifying pain genes to validating known candidates in rodent studies, and to understanding how sex impacts pain processing (see page S7). This is the type of follow-up work that could help researchers to understand the fundamental mechanisms of pain and what the genetic and epigenetic findings have to do with them.

Researchers are not yet close to understanding the genetic components of pain well enough to produce tailored pain therapies to satisfy the push for precision medicine, says Mogil. But he is not pessimistic - just realistic. There is still a lot to learn, and for a researcher that is good news. "What I love about genetics is, you can find interesting proteins to study without even knowing what you're looking for."

Eryn Brown is a freelance writer based in Los Angeles, California.

1. Slade, G. D. et al. J. Pain 14, T116-T124 (2013)

2. Bair, E. et al. Pain 157, 1266-1278 (2016)

3. Bell, J. T. et al. Nature Commun. 5, 2978 (2014) 